\title{
Frequency domain analysis of transmission zeroes on high-speed interconnects in the presence of an orthogonal metal grid underlayer
}

\author{
Yves Quéré, Thierry Le Gouguec, Pierre-Marie Martin, Denis Le Berre, Fabrice Huret \\ Laboratoire d'Electronique et des Systèmes de Télécommunications (LEST) UMR CNRS n6165 \\ 6 Av Le Gorgeu CS93837-29238 BREST Cedex 3 FRANCE \\ Yves.quere@univ-brest.fr; Thierry.LeGouguec@univ-brest.fr .
}

\begin{abstract}
This paper addresses high-speed interconnects in high density systems (Systems on Chip (SoC) in package (SiP) ...). These lines (of miscostrip or coplanar type) often have an underlayer of orthogonal metal grids which can affect transmission characteristics. We subsequently present a characterization through S-parameter measurements and electromagnetic simulations. Two kinds of grid are studied; grounded $(C C)$ and floating grid $(C O)$. In both cases, transmission zeroes appear. The position of these transmission zeroes in the frequency domain depends mainly on the grid length and, of course, on the grid charge $C C$ or $C O$. In order to easily estimate it, we propose a simple equivalent circuit model which we validate by measurements and electromagnetic simulations. We then determine a set of expressions based on this model enabling us to analytically pinpoint the location transmission zero in the frequency domain, valid for any underlayer of orthogonal metal lines or grids.
\end{abstract}

\section{Introduction}

Systems on chip (SoC) and systems in package (SiP) are currently undergoing a period of sustained development. For the SoC, these systems embed on the same chip, analogue functions, RF applications and numerical circuits in order to increase compactness and functionality. The various blocks are connected between them by interconnects realized in a complex 3D medium. With rising operating frequencies in silicon-based integrated circuits, the behavior of on-chip interconnects is becoming increasingly important in overall circuit performance. Transmission line effects need to be taken into account due to the long interconnect line lengths and high frequencies encountered [1]. Other concerns are interconnect line dispersion and losses [2]. Metal lines with simple geometrical configurations, such as single conductor lines, microstrip lines and coplanar waveguide (CPW) [3] have been the main vehicles for characterizing and modelling high-speed interconnect transmission line effects.

The conventional Manhattan routing practice [4] creates on-chip grids by placing two adjacent metal layers perpendicular to each other. It has been shown that the meshes have significant effects on the embedded transmission line characteristics [5][6]. Thus, the effects of orthogonal grids on the transmission characteristics of interconnect lines need to be characterized and modeled.

In this paper, we study the propagation on microstrip or coplanar-like interconnects placed on the higher levels also referred to as "global levels" and above an orthogonal metal grid. We first present structures, measurements and a fullwave electromagnetic analysis of microstrip and coplanar lines situated above a metal grid. We highlight the appearance of a resonance frequency which corresponds to a transmission zero. We than propose a simple circuit model useful for resonant frequency prediction. This model is validated by comparison with measurements and full-wave electromagnetic analysis. Finally we propose analytical expressions allowing the prediction of transmission zero frequencies.

\section{Measurements and electromagnetic analysis.}

Fig. 1 shows the microstrip and coplanar structures with grounded or floating grids respectively. The lines were implemented in multilayer technology available in our laboratory.

In the microstrip case, the lines of width $W=30 \mu \mathrm{m}$ are placed over a substrate thickness $H=60 \mu \mathrm{m}$ and permittivity $\varepsilon_{R}=4.3$. The grids are located in the middle of the substrate and the lower part of the latter is a grounded plane. We have produced several grid densities, subsequently referred to as low density $\left(W_{G}=30 \mu m, S_{G}=110 \mu m\right)$, medium density $\left(W_{G}=30 \mu \mathrm{m}, S_{G}=50 \mu \mathrm{m}\right)$ and large density $\left(W_{G}=90 \mu \mathrm{m}\right.$, $\left.S_{G}=40 \mu m\right)$.

In the case of coplanar structures, there is no grounded plane, but a support in alumina $\left(H_{S}=254 \mu \mathrm{m}\right.$ and $\left.\varepsilon_{R}=9.6\right)$ under the first substrate. The dimensions of the CPW line are: width of ground $W_{M}=270 \mu \mathrm{m}$, space between ground and central conductor $S=70 \mu \mathrm{m}$ and width of the central conductor $W_{C}=30 \mu \mathrm{m}$. The grids have the same characteristics as in the microstrip case.

In general, $L_{G}$ denotes the length of the grid.
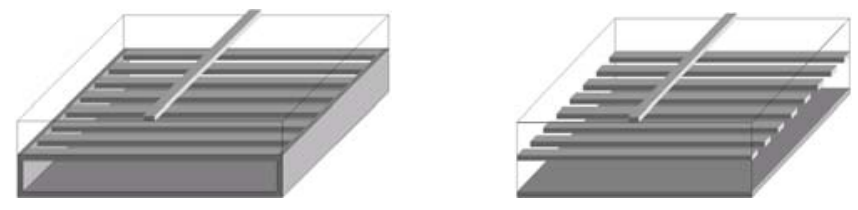

Fig. 1-a: microstrip lines
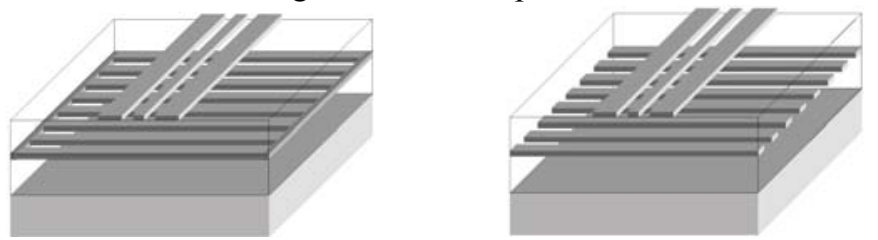

Fig 1-b: Coplanar lines

Fig. 1. Realized microstrip and coplanar test structures 
We present, some photographs of the connected and floating grids in Fig. 2.
Grounded grid

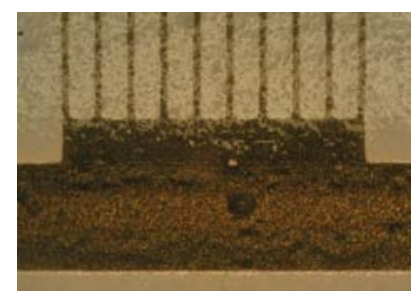

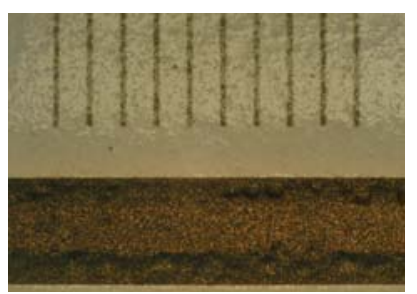

Floating grid
Fig. 2. Photographs of the test structures.

Several microstrip or CPW lines with no grid were also implemented on the same plates as the test structures to serve as refferences. All lines have been provided with coplanar access points allowing probe measurements. The measurements have been performed on a TEKTRONIC networks analyzer in the 1 to $50 \mathrm{GHz}$ band after "TRL" (Thru Reflect Line) calibration.

We present the measured S-parameters of microstrip or coplanar lines in Fig. 3 and Fig. 4, with the orthogonal metal grid underlayer, grounded and, respectively, floating. The corresponding electromagnetic analysis performed with a fullwave Finit Element (FE) code (CAnsoft HFSS) is also presented on the figures.

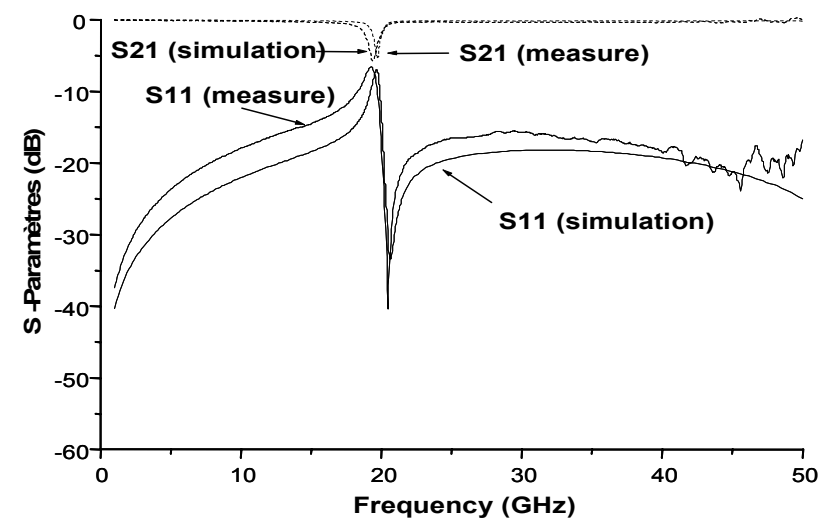

Fig. 3a. S-parameters for microstrip line with grounded grid. ( $W_{G}=30 \mu \mathrm{m}, S_{G}=50 \mu \mathrm{m}, L_{G}=3285 \mu \mathrm{m}$.)

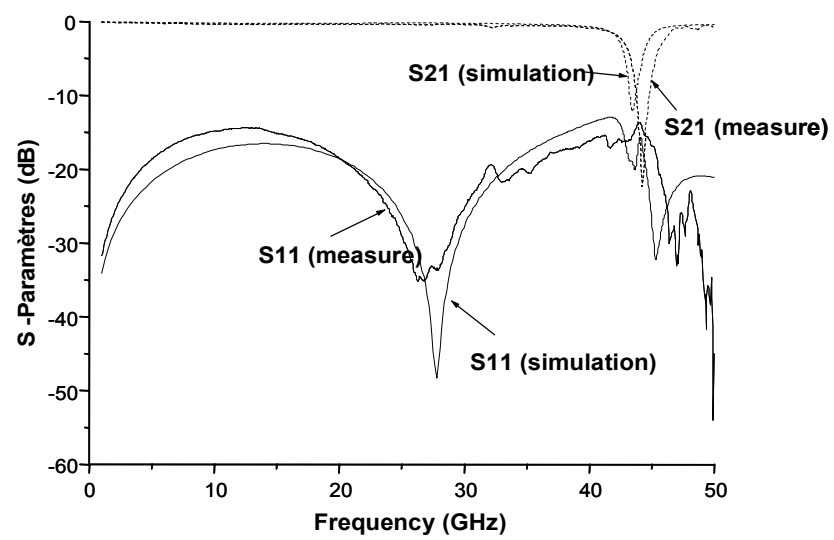

Fig. 3b. S-parameters for microstrip line with floating grid. $\left(W_{G}=30 \mu \mathrm{m}, S_{G}=50 \mu \mathrm{m}, L_{G}=3285 \mu \mathrm{m}\right)$

Fig. 3. Comparison of S-parameters measurement and FE simulations for microstrip structures

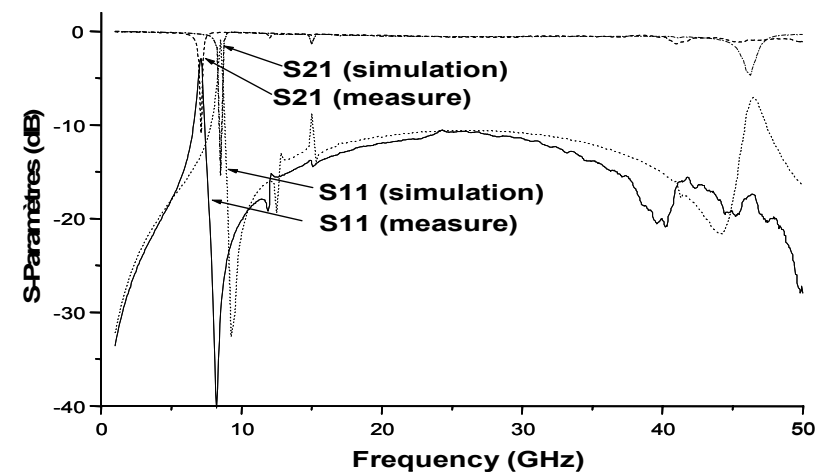

Fig. 4a. S-parameters for coplanar line with grounded grid.

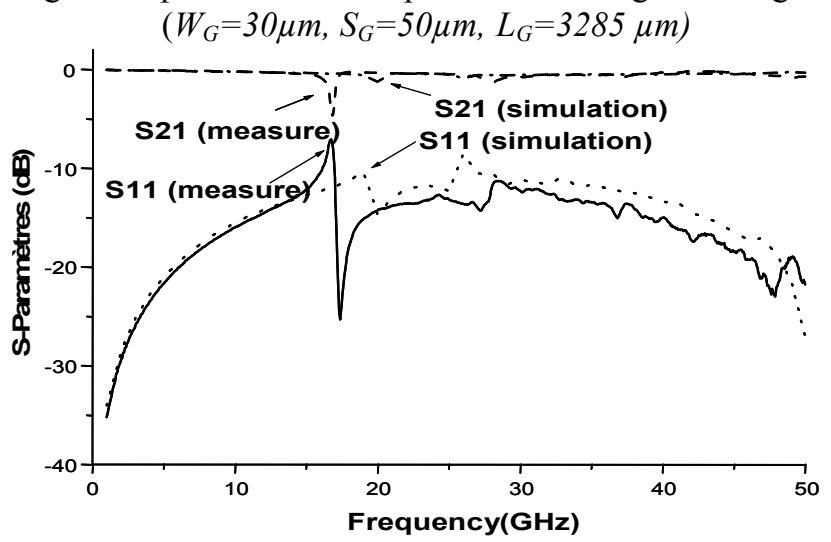

Fig. 4b. S-parameters for coplanar line with floating grid. $\left(W_{G}=30 \mu \mathrm{m}, S_{G}=50 \mu \mathrm{m}, L_{G}=3285 \mu \mathrm{m}\right)$

Fig. 4. Comparison of S-parameters measurement and FE simulations for coplanar structures.

We first notice a good match between measurement and HFSS results, the small differences are probably due to uncertainties in the realization process. This validates the subsequent use of the full-wave electromagnetic simulator to study more structures and elaborate simple and efficient models of lines with orthogonal metal grid underlayer.

In all cases (microstrip or coplanar, with grounded or floating grid), a transmission zero appears for the S21 parameter in a certain frequency point. Models enabling the prediction of such transmission zeroes, that could prove very penalizing on signal propagation, need to be developed. To illustrate this, we have used the S-parameter measurement of the coplanar line with a connected grid underlayer, and we have made a time domain simulation. We present, the simulation principle in Fig. 5, the comparison of the S21 measured parameter and the input signal spectrum in Fig. 6, and the time domain simulation results in Fig. 7

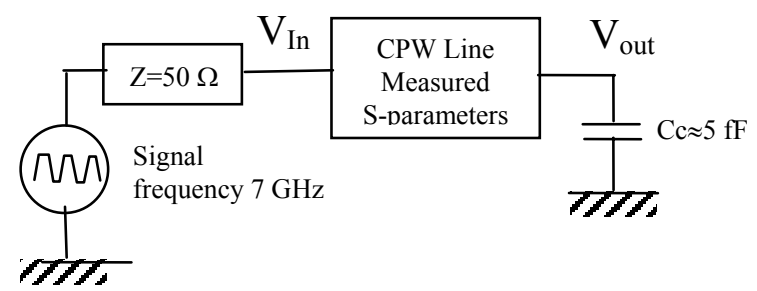

Fig. 5. Schematic's principle for time domain simulation. 


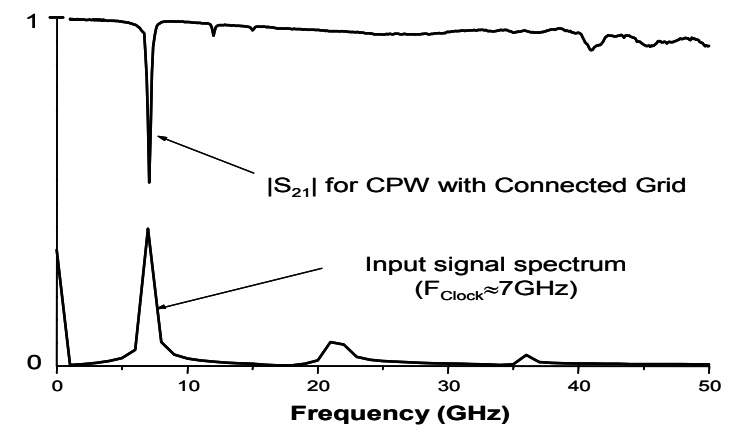

Fig. 6. $\left|S_{21}\right|$ of $C P W$ with connected grid and input signal spectrum.

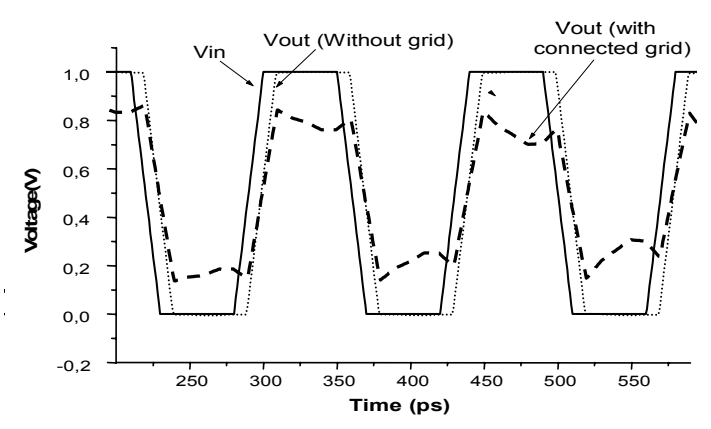

Fig. 7. Time domain results

We note that the location of the transmission zero is not very sensitive to grid density; it mainly seems to depend on the length $L_{G}$ of the grid. In Fig. 8, we present the value of the resonance frequencies versus the length $L_{G}$ for the microstrip line and with both grounded and floating grid respectively. These values are obtained through electromagnetic full-wave simulations (CAnsoft HFSS).

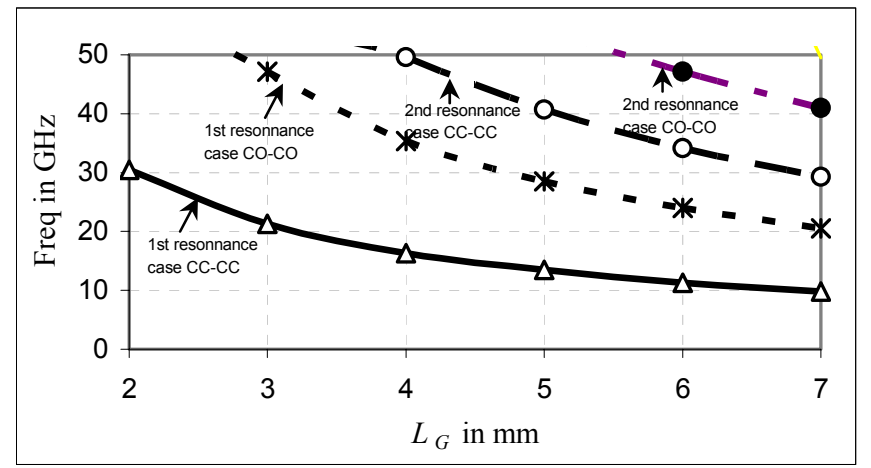

Fig. 8. Resonance frequency versus the length of the grid for the microstrip structure.

\section{Modelling and analysis.}

Seeking to predict resonances frequencies, we sought to develop a simple circuit model describing the behavior of lines over a metal grid. As grids are homogenous, we can study, only one elementary cell at a first approach [7]. We present the concept of the basic cell for microstrip and coplanar structures in Fig. 9. To model a microstrip unit cell, we propose a general equivalent circuit using ideal transmission lines and lumped passive capacitance. The microstrip unit cell is modeled by a line of length $L=S_{G}$, with mid coupling to the grid line through a capacitance $C_{C}$. Two impedances $Z_{\mathrm{CH} 1}$ and $Z_{\mathrm{CH} 2}$ form the grid line's extremities. It follows that $Z_{C H i} \approx 0$ in the case of a connected and $Z_{C H i} \approx \infty$ for the floating grid. The lengths of grid line on either side of the upper microstrip line can differ and they are subsequently denoted as $L_{G 1}$ and $L_{G 2}$. This general unit microstrip cell model is presented Fig. 10.

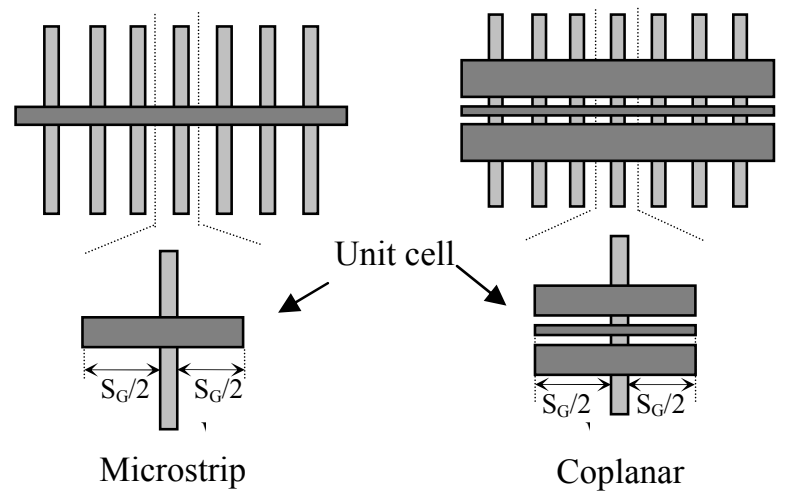

Fig. 9. Definition of a unit cell for microstrip with underlayer orthogonal metal grid

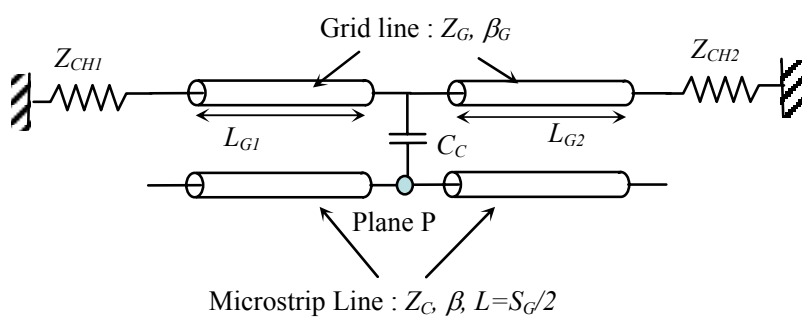

Fig. 10. Modelling of a microstrip unit cell

In figure $10, Z_{C}$ denotes the characteristic impedance of the microstrip line without a grid, and $\beta$ the propagation factor. $Z_{G}$ and $\beta_{G}$ are respectively, the characteristic impedance and the propagation factor of the grid line. All these parameters can easily be determined. The capacitance $C_{C}$ value can be evaluated by using the plane capacitance formula:

$$
C_{C}=\varepsilon_{0} \varepsilon_{R} \frac{W \cdot W_{G}}{H / 2}
$$

$W$ and $W_{G}$ respectively denote the widths of the upper microstrip line and of the grid line. $H$ is the thickness of the substrate, $\varepsilon_{R}$ the relative permittivity and $\varepsilon_{0}$ the void permittivity.

In Fig. 11 we present a comparison between the HFSS results and those obtained by circuit simulation using the unit cell previously described. This case corresponds to a microstrip line over a metal grid, grounded on one side and floating on the other. The grid dimensions are $W_{G}=80 \mu \mathrm{m}$, $S_{G}=70 \mu \mathrm{m}$ and the length $L_{G}=3500 \mu \mathrm{m}$. We can see a good match between the resonance frequencies for the two approaches. 
It should be noted that only resonance frequencies are of interest us, this is why we did not seek to otherwise improve our model.

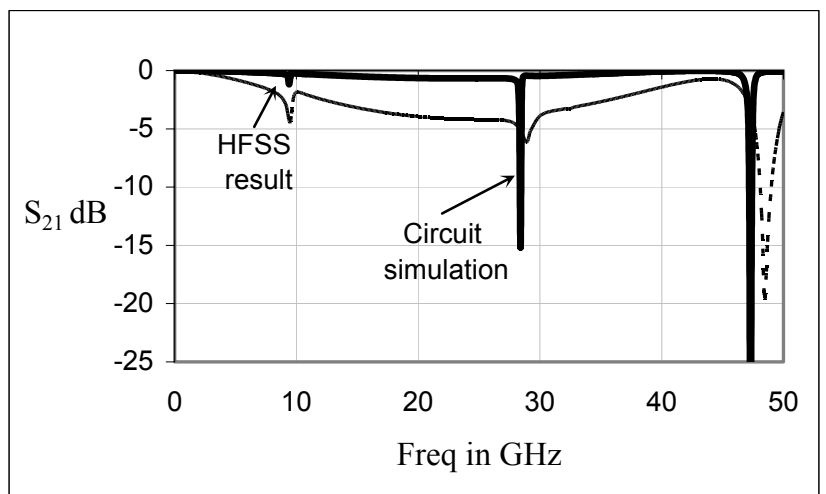

Fig. 11. Comparison between electromagnetic and circuit simulations in case of a grid grounded in one side and floating on the other $\left(W_{G}=80 \mu \mathrm{m}, S_{G}=70 \mu \mathrm{m}, L_{G}=3500 \mu \mathrm{m}\right)$.

The coplanar model is slightly different from the microstrip one, it is presented Fig. 12. Two inductances $L_{\text {ind }}$ have been introduced to simulate the part of the grid without the ground plane above. A more detailed presentation will be made available by the authors during the conference.

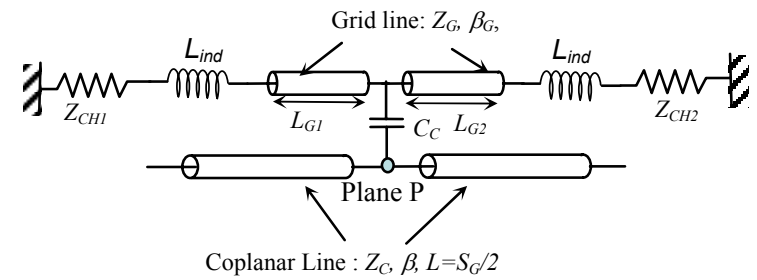

Fig. 12. Modelling of a coplanar unit cell

\section{Computation of the microstrip resonance frequency.}

The use of the simplified model enables us to determine the resonance frequencies which involve a transmission zero.

The impedance $Z_{P}$ seen by the microstrip line in the plane $\mathrm{P}$ (see fig 7) can in a general case be expressed as:

$$
Z_{P}=\frac{1}{j C_{C} \omega}+\frac{Z_{G C 1} \cdot Z_{G C 2}}{Z_{G 1}+Z_{G 2}}
$$

where $Z_{G C l}$ and $Z_{G C 2}$ are respectively the left and right impedances transferred by the grid line to the same point as the capacitance coupling $C_{C}$. These impedances are given by:

$$
Z_{G C i}=Z_{G} \frac{Z_{C H(i)}+j Z_{G} \tan \left(\beta_{G} \cdot L_{G(i)}\right)}{Z_{G}+j Z_{C H(i)} \tan \left(\beta_{G} \cdot L_{G(i)}\right)}
$$

where $Z_{G}$ and $\beta_{G}$ are the propagation characteristics of the grid line, $Z_{C H(i)}$ denotes the impedances on the grid line extremities (for a connected grid $\left(Z_{C H i} \approx 0\right)$ and for a floating grid $\left(Z_{C H i}\right.$ $\approx \infty)$ ). $L_{G(i)}$ is the length of the left or right part of the grid line.

If the microstrip line is short-circuited in plane $\mathrm{P}$, a transmission zero will occur. Consequently, the frequencies where transmission zeros appear are the solutions to equation 4.

$$
Z_{P}=0 \text {. }
$$

In the case where both sides of the grid are connected to the ground the two $Z_{C H(i)}$ impedances are negligible $\left(Z_{C H(i)} \approx 0\right)$ and the expression of the $Z p$ impedance therefore becomes:

$$
Z_{P}=\frac{-j}{C_{C} \omega}+j Z_{G} \frac{\tan \left(\beta_{G} L_{G 1}\right) \tan \left(\beta_{G r i d} L_{G 2}\right)}{\tan \left(\beta_{G} L_{G 1}\right)+\tan \left(\beta_{G} L_{G 2}\right)} .
$$

In the case of the floating grid the two load impedances tend to infinity $\left(Z_{C H(i)} \approx \infty\right)$ and then the $Z p$ impedance can be written as:

$$
Z_{P}=\frac{-j}{C_{C} \omega}+j Z_{G} \frac{1}{\tan \left(\beta_{G} L_{G 1}\right)+\tan \left(\beta_{G} L_{G 2}\right)} .
$$

In the more general case of a grid grounded on one side and floating on the other, the impedance in plane $\mathrm{P}$ can be calculated by:

$$
Z_{P}=\frac{-j}{C_{C} \omega}+j Z_{G} \frac{\tan \left(\beta_{G} L_{G 1}\right)}{1-\tan \left(\beta_{G} L_{G 1}\right) \cdot \tan \left(\beta_{G} L_{G 2}\right)}
$$

\section{Conclusions}

In this paper, we highlight the appearance of transmission zeros for lines situated above an orthogonal metal grid. These transmission zeros can be very penalizing on signal propagation. We consequently propose a simple circuit model to predict the frequencies involving zeros. This simple model based on a circuit approach can be very interesting for VLSI circuit designers. We also translated the circuit model into equations to avoid simulations and to enable rapid analytical computation of orthogonal metal grid critical lengths.

\section{References}

[1] A. Deutsch, and al, "When are transmission-line effects important for on-chip interconnections," in Proc. 47th Electronic Components Technol. Conf.,San Jose, CA, 1997, pp. 704-712.

[2] P. Bhartia and P. Pramanick, "A new microstrip dispersion model," IEEE Trans. Microwave Theory Tech., vol. MTT32, pp. 1379-1384, Oct. 1984.

[3] H. Hasegawa and M. Furukawa, "Properties of microstrip line on Si-SiO2 systems," IEEE Trans. Microwave Theory Tech., vol. MTT-19, pp. 869-881, Nov. 1971.

[4] J. M. Rabaey, "Digital Integrated Circuits: A Design Perspective". Englewood Cliffs, NJ: Prentice-Hall, 1996.

[5] Pingshan Wang and Edwin Chih-Chuan Kan,"High-Speed Interconnects With Underlayer Orthogonal Metal Grids" IEEE Trans on Advanced Packaging, vol. AP-27, $\mathrm{N}^{\circ} 3$, Aug 2004 pp 497-507

[6] Y. Quéré, and al, "High-Frequency Effects of Orthogonal Interconnect Layers on Inductance in High-Speed VLSI Circuits", in Proc 10th IEEE Workshop on Signal Propagation On Interconnects, Berlin-Mitte, Germany, 2006, pp. 253-256.

[7] Richard D. Lutz, and al "Enhanced Transmission Characteristics of On-Chip Interconnects with Orthogonal Gridded Shield" IEEE Trans on Advanced Packaging, vol. AP-24, N³, Aug 2001 pp288-293 